\title{
The Effect of Transcutaneous Electrical Acupoint Stimulation on Inflammatory Response in Patients Undergoing Limb Ischemia-Reperfusion
}

\author{
Yunchang Mo, Sijia Chen, Lili Yang, Ledan Huang, Dan Jin, Zhi Yu, Leilei Wang, \\ Liangrong Wang, Shan Luo, and Junlu Wang \\ Department of Anesthesia, The First Affiliated Hospital of Wenzhou Medical University, Wenzhou, Zhejiang 325000, China \\ Correspondence should be addressed to Junlu Wang; wangjunlu973@163.com
}

Received 2 February 2017; Revised 28 May 2017; Accepted 15 June 2017; Published 19 July 2017

Academic Editor: Alex Kleinjan

Copyright (C) 2017 Yunchang Mo et al. This is an open access article distributed under the Creative Commons Attribution License, which permits unrestricted use, distribution, and reproduction in any medium, provided the original work is properly cited.

\begin{abstract}
Reperfusion after tourniquet use can induce inflammation and cause remote organ injury. We evaluated the therapeutic effect of transcutaneous electrical acupoint stimulation (TEAS) on inflammatory mediators and lung function in patients receiving lower limb tourniquets. Forty patients undergoing unilateral lower extremity surgery with tourniquet were randomly assigned to two groups: the TEAS group and ischemia-reperfusion (I/R) group. The C-C motif chemokine ligand 2 (CCL2), C-X-C motif chemokine ligand 8 (CXCL8), interleukin-1 (IL-1), interleukin-6 (IL-6), interleukin-10 (IL-10), tumor necrosis factor- $\alpha$ (TNF- $\alpha$ ), and arterial blood gas analysis were measured preoperatively and $6 \mathrm{~h}$ after tourniquet removal. The levels of CXCL8, IL-1, IL-6, TNF- $\alpha$, and CCL2 were significantly increased compared to baseline values in both groups, but the increase was significantly smaller in the TEAS group. In the TEAS group, the partial pressure of oxygen and arterial-alveolar oxygen tension ratio were significantly decreased, and the alveolar-arterial oxygen tension difference and respiratory index were significantly increased, compared to those in the I/R group at $6 \mathrm{~h}$ after reperfusion. In conclusion, TEAS diminished the upregulation of proinflammatory factors in response to lower limb ischemia-reperfusion and improved pulmonary gas exchange.
\end{abstract}

\section{Introduction}

Lower limb ischemia can be caused by a variety of clinical conditions, including critical limb ischemia, abdominal aortic aneurysm, and traumatic arterial injury [1]. Therapies that can restore perfusion to the ischemic limb are performed to reduce the injury caused by ischemia. However, reperfusion of the ischemic limb can induce inflammation and cause remote organ injury [2]. The lungs are some of the most vulnerable organs to insult subsequent to ischemia-reperfusion [3-5], which has been proved by models and clinical tests [6-8].

Tourniquets are typically used in orthopedic surgery to reduce intraoperative blood loss and decrease the allogeneic blood transfusion rate, thus providing clear vision for the surgery. This condition may be much more important in orthopedic procedures in which tourniquetrelated ischemia-reperfusion damage develops and the lung gets injured [9]. Our previous research showed that tourniquet-induced lung injury was correlated with impaired gas exchange and that inflammation factors such as interleukin-1 (IL-1), interleukin-6 (IL-6), and tumor necrosis factor- $\alpha$ (TNF- $\alpha$ ) are important mediators of this effect [10]. This suggests that reducing the inflammatory response should have a positive effect on postoperative recovery. The inflammatory factors are released as a result of the impairment of endothelial function and neutrophil infiltration during deflation of the tourniquet [8].

Acupuncture has been proven to exert anti-inflammatory effects in some diseases, such as asthma [11], rhinitis [12], and inflammatory bowel disease [13]. Acupuncture can treat these diseases by reducing the release of inflammatory 


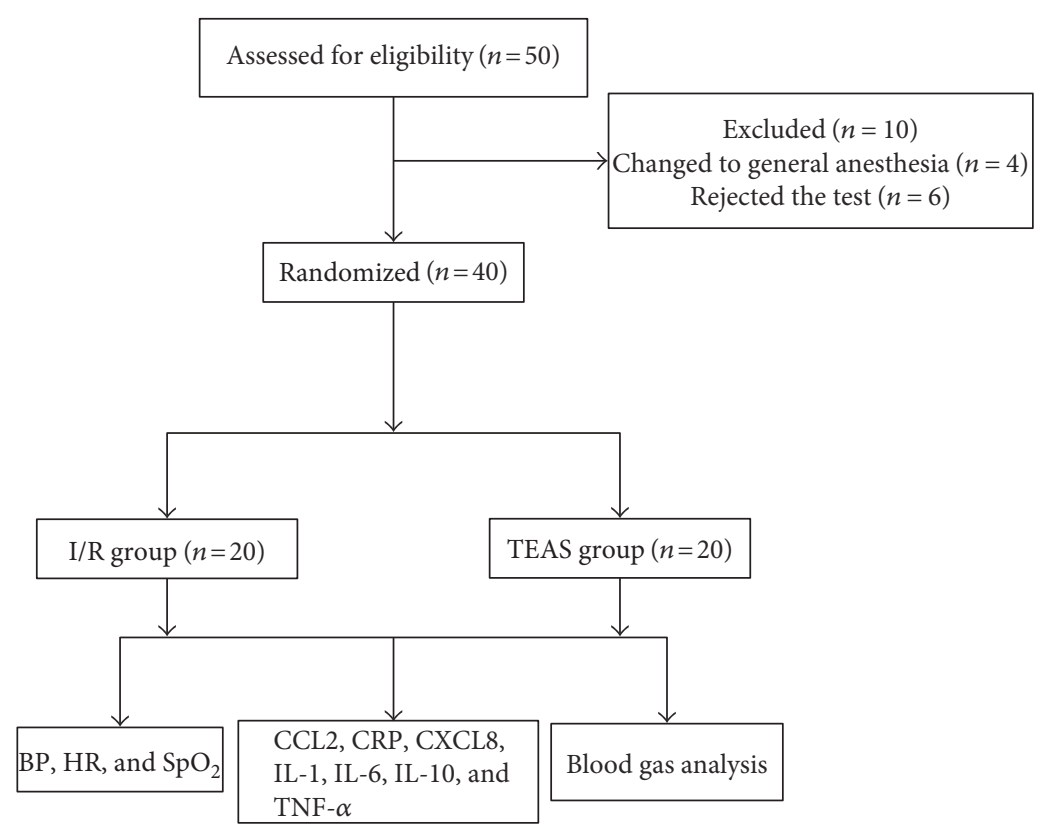

FIGURE 1: Flowchart of study design. I/R: ischemia-reperfusion, TEAS: transcutaneous electrical acupoint stimulation, BP: noninvasive blood pressure, $\mathrm{HR}$ : heart rate, $\mathrm{SpO}_{2}$ : capillary oxygen saturation, CCL2: C-C motif chemokine ligand 2, CRP: C-reactive protein, CXCL8: C-X-C motif chemokine ligand 8, IL-1: interleukin-1, IL-6: interleukin-6, IL-10: interleukin-10, and TNF- $\alpha$ : tumor necrosis factor- $\alpha$.

substances. However, the anti-inflammatory action of acupuncture has not yet been confirmed by large randomized trials. For instance, Tian et al. demonstrated that acupuncture stimulation at ST-36 decreased the production of TNF- $\alpha$ in rats with ulcerative colitis [14]. Acupuncture preconditioning also has certain protective effects on different organs; in particular, its anti-inflammatory effects can protect against lung injury [15]. Song et al. also reported that acupuncture stimulation at ST-36 could reduce severe thermal injury-induced remote acute lung injury in rats [16].

To date, the question of whether transcutaneous electrical acupoint stimulation (TEAS) can protect lung tissue against the adverse effects of ischemia-reperfusion of the lower limbs remains unstudied. In this study, we administered TEAS to lower extremity surgery patients with tourniquets, observed the resulting changes in the inflammatory response, and assessed the pulmonary function by blood gas analysis.

\section{Patients and Methods}

2.1. Study Design and Patient Groups. This randomized, double-blind, placebo-controlled clinical study (clinical trial registration number: ChiCTR-INR-17010499) was approved by the Ethics Committee of the First Affiliated Hospital of Wenzhou Medical University. All participants were informed of the study purpose and design and provided written consent prior to study enrolment. Initially, 50 lower limb surgery patients were selected. They were aged 20 to 65 years old, were of the American Society of Anesthesiologists (ASA) classes I-II, and tourniqueted for 60-90 minutes. The exclusion criteria were as follows: cardiopulmonary dysfunction; taken oxidant and antioxidant drugs within 1 week before surgery; and refused spinal and epidural anesthesia. Ten patients were excluded because of these criteria (Figure 1). Using a random data table, the remaining 40 patients were divided equally into the ischemia-reperfusion group (I/R group) and the transcutaneous electrical acupoint stimulation group (TEAS group).

2.2. TEAS Protocol. The acupoints ST36 and SP6 were chosen in the TEAS group. The TEAS was started at 30 minutes preoperatively and continued until the end of surgery. The parameters for TEAS were as follows: dilatational wave, $2 / 15 \mathrm{~Hz}$, and strength, maximum tolerable intensity. The I/R group received acupuncture at the same acupoints but did not receive stimulation.

2.3. Anesthesia. The patients underwent routine preoperative preparation, including fasting for at least $8 \mathrm{~h}$, establishing venous access, and regular monitoring of the electrocardiogram (ECG), noninvasive blood pressure (BP), heart rate $(\mathrm{HR})$, and peripheral capillary oxygen saturation $\left(\mathrm{SpO}_{2}\right)$. Patients were anesthetized by combined spinal and epidural block performed in L3-4 with $3 \mathrm{~mL}$ of $0.5 \%$ bupivacaine. The block was maintained at approximately the level of T8 for the duration of the surgery. To maintain circulatory stability, Ringer's lactate solution and hydroxyethyl starch injection were infused during the operation. Postoperative infusion of Ringer's lactate solution was performed to meet physiological requirements.

2.4. Data Collection. The mean arterial pressure (MAP) and heart rate (HR) were recorded before anesthesia (T0), $5 \mathrm{~min}$ after anesthesia (T1), $1 \mathrm{~min}$ before removing the tourniquet (T2), and at $1 \mathrm{~min}$ (T3), $5 \mathrm{~min}$ (T4), and $6 \mathrm{~h}$ (T5) after 
TABle 1: Clinical characteristics of the patients ( $n=20$ in each group). Data are expressed as mean \pm standard deviation.

\begin{tabular}{lcccccc}
\hline Group & Gender $(\mathrm{M} / \mathrm{F})$ & Age $(\mathrm{y})$ & Height $(\mathrm{cm})$ & Weight $(\mathrm{kg})$ & Operation time $(\mathrm{min})$ & Tourniquet duration $(\mathrm{min})$ \\
\hline I/R & $13 / 7$ & $42 \pm 12.4$ & $168 \pm 5.9$ & $61.6 \pm 7.8$ & $91.5 \pm 14.7$ & $79.5 \pm 10.1$ \\
TEAS & $12 / 8$ & $43.9 \pm 14.4$ & $165.6 \pm 7.2$ & $63.3 \pm 8.5$ & $90.4 \pm 20.0$ & $80.7 \pm 11.7$ \\
\hline
\end{tabular}

I/R: ischemia-reperfusion; TEAS: transcutaneous electrical acupoint stimulation.

removing the tourniquet. In addition, a $2 \mathrm{~mL}$ radial artery blood sample was collected at T0 and T5: $1 \mathrm{~mL}$ for the gas analysis and the other $1 \mathrm{~mL}$ for the immunoassay (ELISA).

2.5. Blood Gas Analysis. The radial artery blood gas analysis measured the arterial partial pressure of oxygen $\left(\mathrm{PaO}_{2}\right)$ and carbon dioxide $\left(\mathrm{PaCO}_{2}\right)$, as well as hemoglobin $(\mathrm{Hb})$ content. The derived variables included the arterial-alveolar oxygen tension ratio (a/A ratio), alveolar-arterial oxygen tension difference $\left(\mathrm{A}-\mathrm{aO}_{2}\right)$, and respiratory index (RI).

2.6. Enzyme-Linked Immunosorbent Assay (ELISA). Arterial blood ( $1 \mathrm{~mL}$ ) was collected at T0 and T5. Plasma was separated by centrifugation at $2500 \mathrm{rpm}$ for $10 \mathrm{~min}$ and stored at $-80^{\circ} \mathrm{C}$ for further analysis. The serum concentration of C-reactive protein (CRP), TNF- $\alpha$ (tumor necrosis factor- $\alpha$ ), CCL2 (C-C motif chemokine ligand 2), CXCL8 (C-X-C motif chemokine ligand 8), IL-1 (interleukin-1), IL-6 (interleukin6), and IL-10 (interleukin-10) were measured using a human ELISA kit (Jiancheng Co., Nanjing, China), according to the manufacturer's instructions.

2.7. Statistical Analysis. All statistical analyses were performed using SPSS, version 13.0 (SPSS Inc., IL, USA). The discrete variables were analyzed with the chi-square test, repeated measurements were compared using repeated measures' analysis of variance (ANOVA), and continuous variables were compared using the Student's $t$-test. Data were expressed as mean \pm standard deviation. For all tests, $p<0.05$ was considered statistically significant.

\section{Results}

3.1. Clinical Characteristics of Patients. All forty patients completed the study. There were no significant differences in clinical characteristics between the two groups, including gender, age, height, weight, operation time, and tourniquet duration $(p>0.05$; Table 1$)$.

3.2. Hemodynamic Parameters. As shown in Table 2, there were no significant differences in MAP or HR between the two groups $(p>0.05)$.

3.3. Blood Gas Analysis. In the I/R group, the $\mathrm{PaO}_{2}$ and $\mathrm{a} / \mathrm{A}$ ratio were significantly decreased, and the $\mathrm{A}-\mathrm{aO}_{2}$ and respiratory index were significantly increased at $\mathrm{T} 5$, compared to T0 $(p<0.05)$. At T5, the $\mathrm{PaO}_{2}$ and a/A ratio were significantly higher, and the $\mathrm{A}-\mathrm{aO}_{2}$ and respiratory index were significantly lower in the TEAS group, compared with those in the I/R group ( $p<0.05$; Table 3$)$.

3.4. Inflammatory Cytokine and CRP Levels. As shown in Table 4, in the I/R group, the IL-1, IL-6, and CCL2 levels at T5 were significantly higher, compared with those at T0 $(p<0.05)$. Meanwhile, the levels of IL-1, IL-6, CXCL8, CCL2, and TNF- $\alpha$ were significantly lower in the TEAS group at T5, compared to those in the I/R group at T5 $(p<0.05)$. There was no significant difference in CRP and IL-10 between time points or groups $(p>0.05)$.

\section{Discussion}

In the present study, we demonstrated that limb ischemiareperfusion can lead to (1) a systemic inflammatory response, represented by an increase of proinflammatory factors like IL-6 and CCL2, and (2) impaired gas exchange in the lungs, demonstrated by decreased $\mathrm{PaO}_{2}, \mathrm{~A}-\mathrm{aO}_{2}$, and RI. Importantly, we found that TEAS could mitigate these effects.

Although tourniquets play an important role in orthopedic surgery, there are several potential local and systemic complications associated with tourniquet use, including tissue edema, pain, and injuries of remote sites like the heart, lungs, liver, kidney, brain, and other organs [17]. Lung injury can result from changes in capillary permeability and neutrophil infiltration, which lead in turn to impaired lung function [18]. Using a rat model of ischemia-reperfusion, Kalb et al. found a large amount of neutrophil aggregation in the lungs [19]. Another study using an intestinal ischemia-reperfusion model also found lung injury and a significantly lower a/A ratio [20].

4.1. Blood Gas Analysis. The a/A ratio, $\mathrm{A}-\mathrm{aO}_{2}$, and respiratory index can be used to assess pulmonary gas exchange. We found that the a/A ratio, $\mathrm{A}-\mathrm{aO}_{2}$, and respiratory index were significantly decreased at $6 \mathrm{~h}$ after tourniquet release, implying that gas exchange in the lungs was impaired, similar to our findings from a previous study [10]. In addition, we found that these indices were improved by TEAS.

4.2. Inflammatory Cytokine Levels. The mechanism of remote injury due to ischemia-reperfusion of the limbs, especially in the lungs, has not been fully elucidated. However, the inflammatory response has been implicated as an important mediator of lung injury after limb ischemia-reperfusion. Cell damage and stress lead to the activation of inflammatory cells such as macrophages and neutrophils, which can release a large number of inflammatory cytokines, including IL-1, IL-6, TNF- $\alpha$, and CXCL8 [21]. The CRP was also monitored in all patients, and there was no statistical difference between the two groups before and after the operation, indicating that there was no difference in the inflammatory response between the patient groups.

4.2.1. $I L-1$. IL-1 is an acute inflammatory factor that is involved in the process and regulation of ischemiareperfusion injury. Shih et al. established a mouse model of limb ischemia-reperfusion injury and found that IL-1 mRNA 
TABLE 2: Mean arterial pressure and heart rate of the two groups ( $n=20$ in each group). Data are expressed as mean \pm standard deviation.

\begin{tabular}{lccccccc}
\hline & Groups & T0 & T1 & T2 & T3 & T4 & T5 \\
\hline \multirow{2}{*}{ MAP (mmHg) } & I/R & $96 \pm 7$ & $91 \pm 4$ & $90 \pm 6$ & $89 \pm 6$ & $92 \pm 8$ & $91 \pm 5$ \\
& TEAS & $92 \pm 5$ & $91 \pm 4$ & $92 \pm 4$ & $91 \pm 4$ & $92 \pm 6$ & $92 \pm 4$ \\
\multirow{2}{*}{ HR (bpm) } & I/R & $78 \pm 14$ & $80 \pm 13$ & $77 \pm 11$ & $83 \pm 15$ & $74 \pm 9$ & $76 \pm 9$ \\
& TEAS & $77 \pm 10$ & $77 \pm 9$ & $78 \pm 12$ & $81 \pm 9$ & $76 \pm 11$ & $73 \pm 9$ \\
\hline
\end{tabular}

HR: heart rate; I/R: ischemia-reperfusion; MAP: mean arterial pressure; TEAS: transcutaneous electrical acupoint stimulation.

TABle 3: Blood gas analysis $(n=20$ in each group). Data are expressed as mean \pm standard deviation.

\begin{tabular}{|c|c|c|c|}
\hline & Group & T0 & T5 \\
\hline \multirow{2}{*}{$\mathrm{PCO}_{2}$} & $\mathrm{I} / \mathrm{R}$ & $38.85 \pm 3.06$ & $39 \pm 3.15$ \\
\hline & TEAS & $37.55 \pm 2.93$ & $38.8 \pm 3.27$ \\
\hline \multirow{2}{*}{$\mathrm{PaO}_{2}$} & $\mathrm{I} / \mathrm{R}$ & $92.7 \pm 5.58$ & $81.75 \pm 5.03^{*}$ \\
\hline & TEAS & $93.65 \pm 5.87$ & $86.65 \pm 7.23^{*, \#}$ \\
\hline \multirow{2}{*}{$\mathrm{A}-\mathrm{aO}_{2}$} & $\mathrm{I} / \mathrm{R}$ & $8.47 \pm 5.39$ & $19.23 \pm 7.06^{*}$ \\
\hline & TEAS & $9.14 \pm 6.57$ & $14.13 \pm 7.52^{* \#}$ \\
\hline \multirow{2}{*}{ a/A ratio } & $\mathrm{I} / \mathrm{R}$ & $91.68 \pm 5.21$ & $81.12 \pm 6.58^{*}$ \\
\hline & TEAS & $91.20 \pm 6.29$ & $85.78 \pm 8.58^{*, \#}$ \\
\hline \multirow{2}{*}{ RI } & $\mathrm{I} / \mathrm{R}$ & $9.43 \pm 6.49$ & $24.03 \pm 9.91^{*}$ \\
\hline & TEAS & $10.14 \pm 7.37$ & $16.91 \pm 9.79^{*, \#}$ \\
\hline \multirow{2}{*}{$\mathrm{Hb}$} & $\mathrm{I} / \mathrm{R}$ & $10.88 \pm 1.10$ & $10.555 \pm 0.90$ \\
\hline & TEAS & $10.95 \pm 1.26$ & $10.77 \pm 1.25$ \\
\hline
\end{tabular}

${ }^{*} p<0.05$ versus T0. ${ }^{*} p<0.05$ versus the I/R group. a/A ratio: arterial-alveolar oxygen tension ratio; $\mathrm{A}-\mathrm{aO}_{2}$ : alveolar-arterial oxygen tension difference; $\mathrm{Hb}$ : hemoglobin; I/R: ischemia-reperfusion; $\mathrm{PaCO}_{2}$ : arterial partial pressure of carbon dioxide; $\mathrm{PaO}_{2}$ : arterial partial pressure of oxygen; RI: respiratory index; TEAS: transcutaneous electrical acupoint stimulation.

expression in blood was significantly increased at 4 hours after reperfusion [22]. Our study also found that IL-1 levels were significantly increased at 6 hours after reperfusion in the I/R group and that TEAS could significantly reduce this.

4.2.2. IL-6. Studies have shown that the peripheral blood concentration of IL- 6 is proportional to the degree of ischemia-reperfusion injury; it was a predictor of the severity of tissue damage and was found to correlate with an increasing incidence of complications $[23,24]$. In the present study, IL-6 levels were significantly elevated at $6 \mathrm{~h}$ after tourniquet removal in both groups, but the increase in the TEAS group was significantly less than that in the I/R group.

4.2.3. CXCL8. CXCL8 is also named interleukin-8 (IL-8), which is a proinflammatory response factor that is produced by a variety of cells, such as monocytes, macrophages, and $\mathrm{T}$ cells. CXCL8 levels can be significantly increased after ischemia-reperfusion, likely due to cell damage and stress caused by neutrophils, monocytes, macrophages, and other inflammatory cells. A previous study also confirmed that serum CXCL8 levels were significantly increased at 2, 6, and 24 hours after reperfusion in patients with limb ischemiareperfusion injury [25]. In this study, we also found that CXCL8 levels were elevated at 6 hours after reperfusion,
TABle 4: Plasma CRP, CCL2, CXCL8, IL-1, IL-6, TNF- $\alpha$, and IL-10 levels ( $n=20$ in each group). Data are expressed as mean \pm standard deviation.

\begin{tabular}{lccc}
\hline & Groups & T0 & T5 \\
\hline CRP $(\mathrm{pg} / \mathrm{mL})$ & I/R & $7.9 \pm 1.2$ & $8.4 \pm 1.6$ \\
& TEAS & $7.7 \pm 2.0$ & $8.0 \pm 1.9$ \\
CCL2 $(\mathrm{pg} / \mathrm{mL})$ & I/R & $108.8 \pm 38.8$ & $154.2 \pm 42.9^{*}$ \\
& TEAS & $107.1 \pm 31.0$ & $111.7 \pm 50.0^{\#}$ \\
CXCL8 $(\mathrm{pg} / \mathrm{mL})$ & I/R & $8.1 \pm 3.0$ & $24.2 \pm 6.2^{*}$ \\
& TEAS & $8.5 \pm 3.4$ & $16.3 \pm 4.2^{*, \#}$ \\
IL-1 $(\mathrm{pg} / \mathrm{mL})$ & I/R & $18.6 \pm 4.4$ & $31.4 \pm 9.3^{*}$ \\
& TEAS & $17.7 \pm 4.3$ & $23.9 \pm 7.5^{*, \#}$ \\
IL-6 $(\mathrm{pg} / \mathrm{mL})$ & I/R & $6.1 \pm 2.0$ & $61.6 \pm 4.5^{*}$ \\
& TEAS & $5.4 \pm 1.0$ & $30.4 \pm 4.5^{*, \#}$ \\
TNF- $\alpha(\mathrm{pg} / \mathrm{mL})$ & I/R & $16.7 \pm 6.0$ & $20.8 \pm 8.9$ \\
& TEAS & $17.2 \pm 7.7$ & $12.0 \pm 4.7^{*, \#}$ \\
IL-10 $(\mathrm{pg} / \mathrm{mL})$ & I/R & $2.5 \pm 1.0$ & $2.8 \pm 1.2$ \\
& TEAS & $2.9 \pm 1.2$ & $3.6 \pm 1.3$ \\
\hline
\end{tabular}

${ }^{*} p<0.05$ versus T0. ${ }^{\#} p<0.05$ versus the I/R group. I/R: ischemiareperfusion; CRP: C-reactive protein; CCL2: C-C motif chemokine ligand 2; CXCL8: C-X-C motif chemokine ligand 8; IL-1: interleukin-1; IL-6: interleukin-6; IL-10: interleukin-10; TEAS: transcutaneous electrical acupoint stimulation; TNF- $\alpha$ : tumor necrosis factor- $\alpha$.

and the TEAS group showed significantly lower CXCL8 levels at 6 hours after reperfusion than the I/R group.

4.2.4. TNF- $\alpha$. TNF- $\alpha$ is one of the earliest released and most sensitive inflammation factors [8]. It can induce the production of IL-6, further stimulating the inflammatory response. Anti-TNF antibodies have been shown to reduce pulmonary injury following lower limb ischemia-reperfusion [26, 27]. In the present study, TNF- $\alpha$ levels were significantly higher at $\mathrm{T} 5$ in the I/R group as compared to the TEAS group.

4.2.5. CCL2. CCL2 is a basophil chemotactic and activating agent, that is, it stimulates basophil degranulation and histamine release $[28,29]$. CCL2 is also named monocyte chemotactic protein 1 (MCP-1), which is one factor of the inflammatory responses, and controls the production and release of other inflammatory mediators. Furthermore, CCL2 plays a critical role in attracting a variety of inflammatory cells, especially the mononuclear cells, to gather at affected areas. Abbruzzese et al. showed that CCL2 levels were increased after reperfusion in a mouse model of limb ischemia-reperfusion [30]. As was the case with IL-6 and TNF- $\alpha$, the levels of CCL 2 were increased after reperfusion 
in both groups, but the increase in the TEAS group was significantly less than that in the I/R group.

4.2.6. IL-10. IL-10 is an anti-inflammatory factor that inhibits the production of IL- 6 and TNF- $\alpha$. A moderate amount of IL-10 inhibits the secretion of inflammatory mediators and promotes the release of anti-inflammatory factors [31]. We did not observe any significant differences in IL-10 levels in the present study, suggesting that the effect of TEAS is restricted to proinflammatory agents.

4.3. Effects of Acupuncture and TEAS. Studies have shown that acupuncture functions as a stimulus signal that is transmitted through the sciatic nerve to produce antiinflammatory effects in the central nervous system [32]. Studies also have shown that electric acupuncture at zusanli (ST36) and sanyinjiao (SP6) has anti-inflammatory and organ-protective effects $[16,33]$, which were correlated with vagus nerve excitement. Torres-Rosas et al. found that acupuncture at ST36 reduced the levels of IL-6 and TNF- $\alpha$ in sepsis mice, thus reducing postoperative infection and improving the survival rate [32]. Furthermore, animal experiments and clinical studies have demonstrated that acupuncture at ST36 can reduce the levels of abnormally increased proinflammatory factors during the inflammatory response $[16,34]$.

Acupuncture can also play an important antiinflammatory role in different diseases [13]. In a rat model of neuropathic pain, Cha et al. found that electroacupuncture could reduce the expression of proinflammatory factors and reduce the amount of pain [35]. In other inflammation-related disease models, such as rheumatoid arthritis [36], ulcerative colitis, and achronic stress models of depression [37], acupuncture has also been found to reduce the levels of inflammatory factors and alleviate the disease symptoms [38].

At present, ST36 and SP6 are two of the most recognized acupuncture points with clear anti-inflammatory effects [32]. In the present study, we found that patients who received TEAS at these points had significantly lower levels of IL-6, TNF- $\alpha$, and CCL2 at $6 \mathrm{~h}$ after reperfusion. In addition, we found that IL-10 levels were not different between time points or groups. Guo et al. found a similar result in a rat model of acute pancreatitis [39]. This suggests that TEAS may play a critical role in anti-inflammation through reducing the levels of proinflammatory cytokines in the plasma but may not have much influence on antiinflammatory cytokines.

\section{Conclusions}

Although short-term tourniquet use is generally considered safe, we found that lower extremity surgery patients who received a tourniquet exhibited an increase in the levels of proinflammatory cytokines and impaired lung function. Although there were no complications in the present group, patients with more risk factors, such as the elderly, and those experiencing cardiopulmonary dysfunction, multiple injuries, emergency operation, or a delayed tourniquet duration, might be more susceptible to reperfusion injury. TEAS at ST36 and SP6 was found to mitigate these effects; it is a minimally invasive intervention that can be safely and effectively applied in clinical settings.

\section{Conflicts of Interest}

The authors declare that there is no conflict of interest regarding the publication of this paper.

\section{Authors' Contributions}

Yunchang Mo, Sijia Chen, and Lili Yang contributed equally to this work.

\section{Acknowledgments}

This study was financially supported by the National Natural Science Foundation of China (Grants 81603685, 81273926, and 81573742), Zhejiang Province Natural Science Foundation (Grants LY15H270014 and LY15H290006), and Wenzhou Science and Technology Bureau Foundation (Grants Y20150229 and Y20140706).

\section{References}

[1] M. Volanska, P. Zavacky, and J. Bober, "Ischaemic-reperfusion damage of tissue and critical limb ischaemia," Bratislavské Lekárske Listy, vol. 107, no. 6-7, pp. 264-268, 2006.

[2] M. C. Ott, J. R. Scott, A. Bihari et al., "Inhalation of carbon monoxide prevents liver injury and inflammation following hind limb ischemia/reperfusion," FASEB Journal : Official Publication of the Federation of American Societies for Experimental Biology, vol. 19, no. 1, pp. 106-108, 2005.

[3] M. M. Yassin, D. W. Harkin, D. A. A. Barros, M. I. Halliday, and B. J. Rowlands, "Lower limb ischemia-reperfusion injury triggers a systemic inflammatory response and multiple organ dysfunction," World Journal of Surgery, vol. 26, no. 1, pp. 115121, 2002.

[4] R. M. Roumen, T. Hendriks, J. Ven-Jongekrijgvan der et al., "Cytokine patterns in patients after major vascular surgery, hemorrhagic shock, and severe blunt trauma. Relation with subsequent adult respiratory distress syndrome and multiple organ failure," Annals of Surgery, vol. 218, no. 6, pp. 769776, 1993.

[5] A. B. Groeneveld, P. G. Raijmakers, J. A. Rauwerda, and C. E. Hack, "The inflammatory response to vascular surgeryassociated ischaemia and reperfusion in man: effect on postoperative pulmonary function," European Journal of Vascular and Endovascular Surgery: The Official Journal of the European Society for Vascular Surgery, vol. 14, no. 5, pp. 351-359, 1997.

[6] J. Punch, R. Rees, B. Cashmer, K. Oldham, E. Wilkins, and D. J. Smith, "Acute lung injury following reperfusion after ischemia in the hind limbs of rats," The Journal of Trauma, vol. 31, no. 6, pp. 760-765, 1991, discussion 765-767.

[7] G. Plitas, P. J. Gagne, B. E. Muhs et al., "Experimental hindlimb ischemia increases neutrophil-mediated matrix metalloproteinase activity: a potential mechanism for lung injury after limb ischemia," Journal of the American College of Surgeons, vol. 196, no. 5, pp. 761-767, 2003. 
[8] R. K. Kharbanda, M. Peters, B. Walton et al., "Ischemic preconditioning prevents endothelial injury and systemic neutrophil activation during ischemia-reperfusion in humans in vivo," Circulation, vol. 103, no. 12, pp. 1624-1630, 2001.

[9] L. N. Lin, L. R. Wang, W. T. Wang et al., "Ischemic preconditioning attenuates pulmonary dysfunction after unilateral thigh tourniquet-induced ischemia-reperfusion," Anesthesia and Analgesia, vol. 111, no. 2, pp. 539-543, 2010.

[10] L. Lin, L. Wang, Y. Bai et al., "Pulmonary gas exchange impairment following tourniquet deflation: a prospective, singleblind clinical trial," Orthopedics, vol. 33, no. 6, p. 395, 2010.

[11] C. F. Liu and L. W. Chien, "Efficacy of acupuncture in children with asthma: a systematic review," Italian Journal of Pediatrics, vol. 41, p. 48, 2015.

[12] M. B. Taw, W. D. Reddy, F. S. Omole, and M. D. Seidman, "Acupuncture and allergic rhinitis," Current Opinion in Otolaryngology \& Head and Neck Surgery, vol. 23, no. 3, pp. 216-220, 2015.

[13] F. J. Zijlstra, I. Berg-de Langevan den, F. J. Huygen, and J. Klein, "Anti-inflammatory actions of acupuncture," Mediators of Inflammation, vol. 12, no. 2, pp. 59-69, 2003.

[14] L. Tian, Y. X. Huang, M. Tian, W. Gao, and Q. Chang, "Downregulation of electroacupuncture at ST36 on TNF-alpha in rats with ulcerative colitis," World Journal of Gastroenterology, vol. 9, no. 5, pp. 1028-1033, 2003.

[15] C. L. Huang, C. J. Huang, P. S. Tsai, L. P. Yan, and H. Z. Xu, "Acupuncture stimulation of ST-36 (Zusanli) significantly mitigates acute lung injury in lipopolysaccharide-stimulated rats," Acta Anaesthesiologica Scandinavica, vol. 50, no. 6, pp. 722-730, 2006.

[16] X. M. Song, X. J. Wu, J. G. Li et al., “The effect of electroacupuncture at ST36 on severe thermal injury-induced remote acute lung injury in rats," Burns : Journal of the International Society for Burn Injuries, vol. 41, no. 7, pp. 1449-1458, 2015.

[17] F. Q. Gao, Z. J. Li, K. Zhang, D. Huang, and Z. J. Liu, "Risk factors for lower limb swelling after primary total knee arthroplasty," Chinese Medical Journal, vol. 124, no. 23, pp. 3896-3899, 2011.

[18] H. Anner, R. P. Kaufman, L. Kobzik, C. R. Valeri, D. Shepro, and H. B. Hechtman, "Pulmonary leukosequestration induced by hind limb ischemia," Annals of Surgery, vol. 206, no. 2, pp. 162-167, 1987.

[19] R. Kalb, P. Schober, L. A. Schwarte, J. Weimann, and S. A. Loer, "Preconditioning, but not postconditioning, with Sevoflurane reduces pulmonary neutrophil accumulation after lower body ischaemia/reperfusion injury in rats," European Journal of Anaesthesiology, vol. 25, no. 6, pp. 454459, 2008.

[20] C. Li, Y. S. Li, M. Xu et al., "Limb remote ischemic preconditioning for intestinal and pulmonary protection during elective open infrarenal abdominal aortic aneurysm repair: a randomized controlled trial," Anesthesiology, vol. 118, no. 4, pp. 842-852, 2013.

[21] O. Koksel, C. Yildirim, L. Cinel et al., "Inhibition of poly(ADPribose) polymerase attenuates lung tissue damage after hind limb ischemia-reperfusion in rats," Pharmacological Research, vol. 51, no. 5, pp. 453-462, 2005.

[22] Y. M. Shih, J. M. Shih, M. H. Pai, Y. C. Hou, C. L. Yeh, and S. L. Yeh, "Glutamine administration after sublethal lower limb ischemia reduces inflammatory reaction and offers organ protection in ischemia/reperfusion injury," JPEN.
Journal of Parenteral and Enteral Nutrition, vol. 40, no. 8, pp. 1122-1130, 2016.

[23] V. Asensi, E. Valle, A. Meana et al., "In vivo interleukin-6 protects neutrophils from apoptosis in osteomyelitis," Infection and Immunity, vol. 72, no. 7, pp. 3823-3828, 2004.

[24] S. G. Muehlstedt, C. J. Richardson, M. Lyte, and J. L. Rodriguez, "Systemic and pulmonary effector cell function after injury," Critical Care Medicine, vol. 30, no. 6, pp. 13221326, 2002.

[25] L. Wang, W. Wang, X. Zhao et al., "Effect of Shenmai injection, a traditional Chinese medicine, on pulmonary dysfunction after tourniquet-induced limb ischemia-reperfusion," The Journal of Trauma, vol. 71, no. 4, pp. 893-897, 2011.

[26] R. Welbourn, G. Goldman, M. O'Riordain et al., "Role for tumor necrosis factor as mediator of lung injury following lower torso ischemia," Journal of Applied Physiology, vol. 70, no. 6, pp. 2645-2649, 1991.

[27] P. Sorkine, A. Setton, P. Halpern et al., "Soluble tumor necrosis factor receptors reduce bowel ischemia-induced lung permeability and neutrophil sequestration," Critical Care Medicine, vol. 23, no. 8, pp. 1377-1381, 1995.

[28] R. Alam, M. A. Lett-Brown, P. A. Forsythe et al., "Monocyte chemotactic and activating factor is a potent histaminereleasing factor for basophils," The Journal of Clinical Investigation, vol. 89, no. 3, pp. 723-728, 1992.

[29] P. Kuna, S. R. Reddigari, D. Rucinski, J. J. Oppenheim, and A. P. Kaplan, "Monocyte chemotactic and activating factor is a potent histamine-releasing factor for human basophils," The Journal of Experimental Medicine, vol. 175, no. 2, pp. 489493, 1992.

[30] T. A. Abbruzzese, H. Albadawi, J. Kang et al., "Enoxaparin does not ameliorate limb ischemia-reperfusion injury," The Journal of Surgical Research, vol. 147, no. 2, pp. 260-266, 2008.

[31] A. Cianciulli, T. Dragone, R. Calvello et al., "IL-10 plays a pivotal role in anti-inflammatory effects of resveratrol in activated microglia cells," International Immunopharmacology, vol. 24, no. 2, pp. 369-376, 2015.

[32] R. Torres-Rosas, G. Yehia, G. Pena et al., "Dopamine mediates vagal modulation of the immune system by electroacupuncture," Nature Medicine, vol. 20, no. 3, pp. 291-295, 2014.

[33] J. W. J. LNDu, G. C. Wu, and X. D. Cao, "Naloxone and electroacunpunture (EA) improve the immune function of traumatized rats," Sheng li Xue Bao : [Acta Physiologica Sinica], vol. 50, no. 6, pp. 636-642, 1998.

[34] A. L. Nager, M. Kobylecka, P. K. Pham, L. Johnson, and J. I. Gold, "Effects of acupuncture on pain and inflammation in pediatric emergency department patients with acute appendicitis: a pilot study," The Journal of Alternative and Complementary Medicine : Research on Paradigm, Practice, and Policy, vol. 21, no. 5, pp. 269-272, 2015.

[35] M. H. Cha, T. S. Nam, Y. Kwak, H. Lee, and B. H. Lee, "Changes in cytokine expression after electroacupuncture in neuropathic rats," Evidence-Based Complementary and Alternative Medicine : eCAM, vol. 2012, Article ID 792765, 6 pages, 2012.

[36] A. M. Attia, F. A. Ibrahim, E. N. A. Abd et al., "Therapeutic antioxidant and anti-inflammatory effects of laser acupuncture on patients with rheumatoid arthritis," Lasers in Surgery and Medicine, vol. 48, no. 5, pp. 490-497, 2016.

[37] J. Lu, R. H. Shao, L. Hu, Y. Tu, and J. Y. Guo, "Potential antiinflammatory effects of acupuncture in a chronic stress 
model of depression in rats," Neuroscience Letters, vol. 618, pp. 31-38, 2016.

[38] H. G. Wu, L. B. Zhou, Y. Y. Pan et al., "Study of the mechanisms of acupuncture and moxibustion treatment for ulcerative colitis rats in view of the gene expression of cytokines," World Journal of Gastroenterology, vol. 5, no. 6, pp. 515-517, 1999.

[39] H. Guo, S. F. Zhu, R. R. Zhang, X. L. Zhao, M. H. Wan, and W. F. Tang, "Electroacupuncture ameliorates acute lung injury through promoting gastrointestinal motility in rats with acute pancreatitis," Evidence-Based Complementary and Alternative Medicine : eCAM, vol. 2014, Article ID 943596, 8 pages, 2014. 


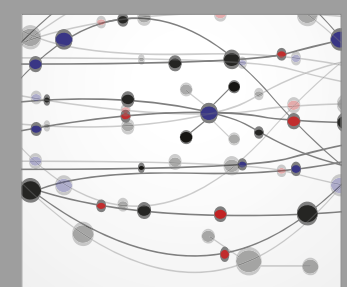

The Scientific World Journal
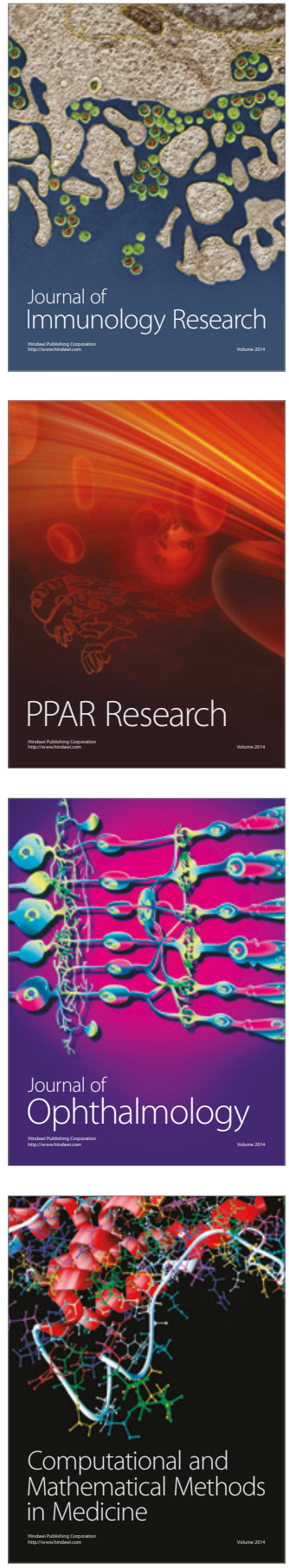

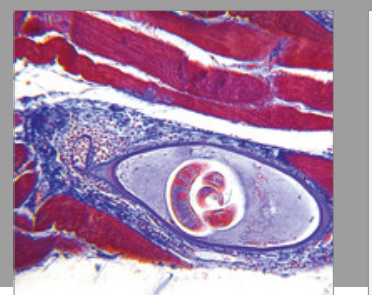

Gastroenterology Research and Practice
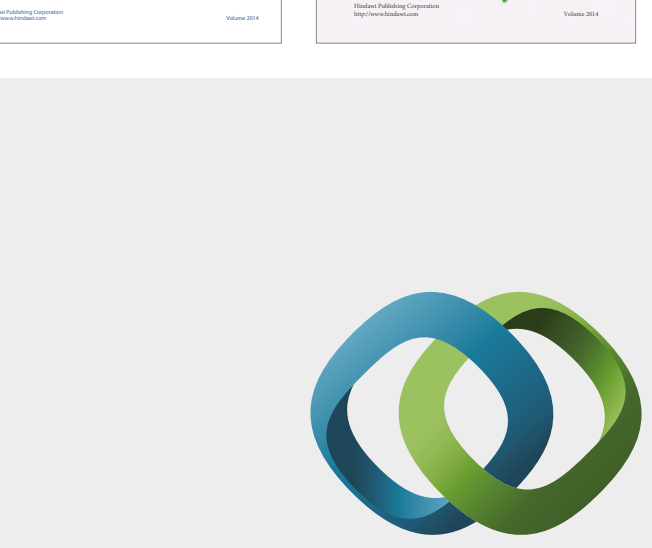

\section{Hindawi}

Submit your manuscripts at

https://www.hindawi.com
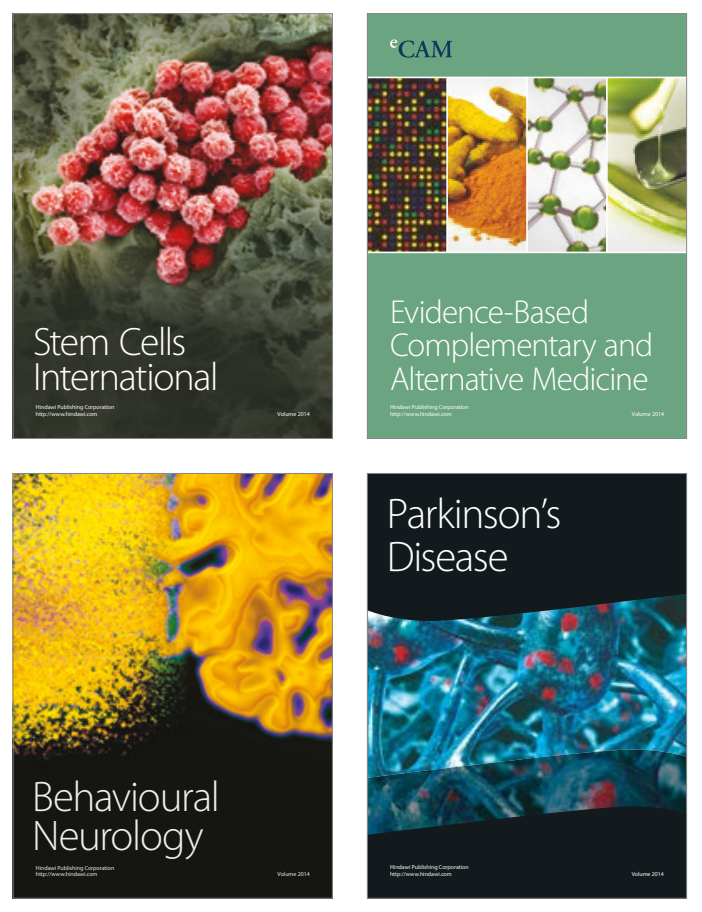
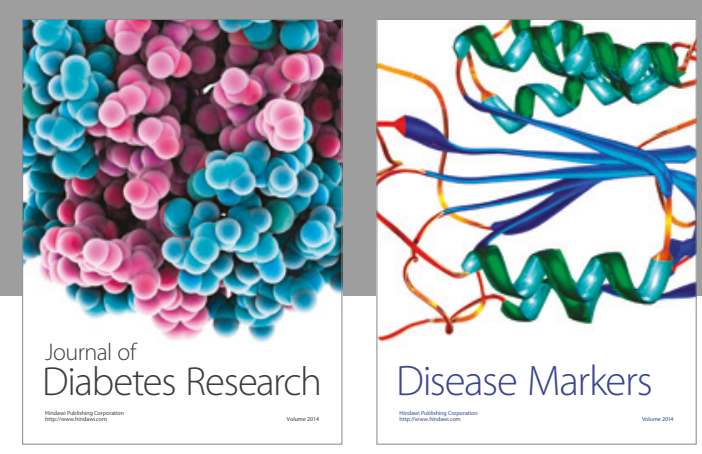

Disease Markers
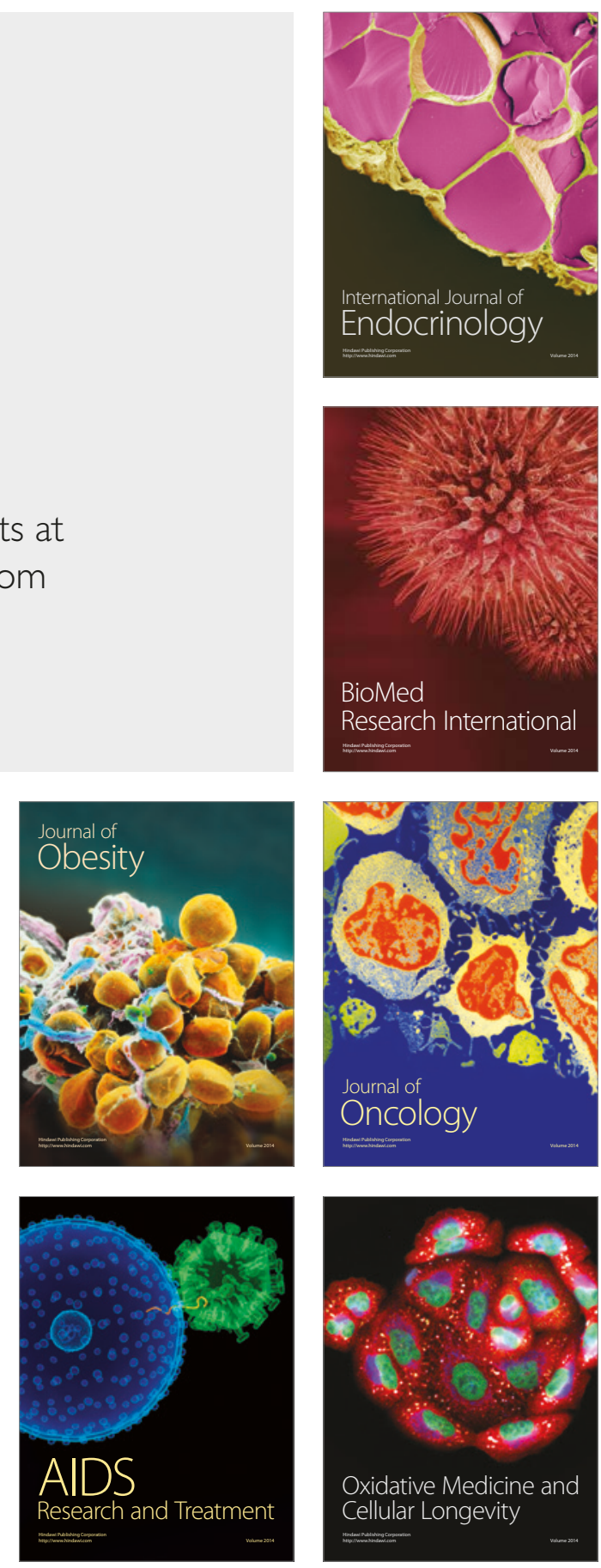